\title{
EVERYWHERE REGULARITY FOR VECTORIAL FUNCTIONALS WITH GENERAL GROWTH
}

\author{
Elvira Mascolo ${ }^{1}$ And Anna Paola Migliorini ${ }^{1}$
}

\begin{abstract}
We prove Lipschitz continuity for local minimizers of integral functionals of the Calculus of Variations in the vectorial case, where the energy density depends explicitly on the space variables and has general growth with respect to the gradient. One of the models is
\end{abstract}

$$
F(u)=\int_{\Omega} a(x)[h(|D u|)]^{p(x)} \mathrm{d} x
$$

with $h$ a convex function with general growth (also exponential behaviour is allowed).

Mathematics Subject Classification. 49N60, 35J50.

Received September 4, 2001.

\section{INTRODUCTION}

In this paper we study the local Lipschitz continuity for local minimizers of the integral functional

$$
F(u)=\int_{\Omega} f(x, D u(x)) \mathrm{d} x
$$

where $\Omega \subset \mathbf{R}^{n}$ is an open set, $f=f(x, \xi): \Omega \times \mathbf{R}^{n N} \rightarrow \mathbf{R}$ is a Carathéodory function and $D u=\left(u_{x_{i}}^{\alpha}\right)$ for $i=1, \ldots, n(n \geq 2)$ and $\alpha=1, \ldots, N$ denotes the Jacobian matrix of the vector-valued function $u: \Omega \rightarrow \mathbf{R}^{N}$.

We say that $u \in W_{\text {loc }}^{1,2}\left(\Omega, \mathbf{R}^{N}\right)$ is a local minimizer of $F$ if $f(x, D u) \in L_{\text {loc }}^{1}(\Omega)$ and for every $\varphi \in C_{0}^{1}\left(\Omega, \mathbf{R}^{N}\right)$

$$
\int_{\operatorname{spt} \varphi} f(x, D u) \mathrm{d} x \leq \int_{\operatorname{spt} \varphi} f(x, D u+D \varphi) \mathrm{d} x
$$

therefore $u$ is also a weak solution of an elliptic system of the form

$$
\sum_{i=1}^{n} \frac{\partial}{\partial x_{i}} a_{i}^{\alpha}(x, D u)=0, \quad \forall \alpha=1, \ldots, N
$$

where the vector field $a=\left(a_{i}^{\alpha}\right): \Omega \times \mathbf{R}^{n N} \rightarrow \mathbf{R}^{n N}$ is the gradient with respect to $\xi$ of the function $f$.

Keywords and phrases. Minimizers, regularity, nonstandard growth, exponential growth.

1 Dipartimento di Matematica "U. Dini", Universita' di Firenze, viale Morgagni 67/A, 50134 Firenze, Italy;

e-mail: mascolo@math.unifi.it 
The regularity properties for minimizers of vectorial integrals have been widely investigated under ellipticity and natural growth conditions and, in general, we can aspect only partial regularity, see [10,12]. Nevertheless, in the case $f(x, \xi)=|\xi|^{p},(p \geq 2)$, Uhlenbeck proved in [23] that the minimizers are in $C_{\text {loc }}^{1, \alpha}\left(\Omega, \mathbf{R}^{N}\right)$. Partial regularity is obtained when integrands have the form $g(x, u,|\xi|)$ with $|\xi|^{p}$ behaviour by Giaquinta and Modica [11] for $p \geq 2$ and Acerbi and Fusco [2] for $1<p<2$.

In the last years the interest in the study of regularity under non natural growth conditions has developed new approaches. In [15] Marcellini considers integrals without growth conditions and proves Hölder continuity of the gradient for minimizers when $f(x, \xi)=g(|\xi|)$ with $g$ positive and convex, satisfying:

$$
\frac{g^{\prime}(t)}{t} \text { is positive and increasing in }(0,+\infty)
$$

and a non oscillatory condition at infinity, i.e. for every $\alpha>1$ there exists a constant $c=c(\alpha)$ such that

$$
g^{\prime \prime}(t) t^{2 \alpha} \leq c[g(t)]^{\alpha}, \quad \forall t>1
$$

these conditions imply at least quadratic growth but they allow exponential behaviour. The subquadratic case is studied by Leonetti et al. [9].

In this paper we consider the non homogeneous case

$$
f(x, \xi)=g(x,|\xi|)
$$

and we obtain the following regularity result:

Theorem 1.1. Let $g=g(x, t): \Omega \times[0,+\infty) \rightarrow[0,+\infty)$ be a function of class $C^{2}$, convex in $t$, such that $\forall x \in \Omega$, $\frac{g_{t}(x, t)}{t}$ is positive and increasing with respect to $t$. Assume that for every $\Omega_{0} \subset \subset \Omega$ and $\alpha>1$ there exist two positive constants $c_{1}$ and $c_{2}$, depending on $\alpha$ and on $\Omega_{0}$, such that $\forall x \in \Omega_{0}$

$$
\begin{gathered}
g_{t t}(x, t) t^{2 \alpha} \leq c_{1}[g(x, t)]^{\alpha}, \quad \forall t \geq 1 \\
\left|g_{t x_{s}}(x, t)\right| \leq c_{2} g_{t}(x, t)\left[1+g_{t}^{\alpha-1}(x, t)\right], \quad \forall t \geq 0, \forall s=1, \ldots, n .
\end{gathered}
$$

Then every local minimizer $u$ of the functional (1.1) with $f$ given by (1.5) is in $W_{\text {loc }}^{1, \infty}\left(\Omega, \mathbf{R}^{N}\right)$ and there exist $c>0$ and $\sigma>0$ such that for every $B_{R} \subset \subset \Omega$

$$
\sup _{B_{R / 2}}|D u| \leq c\left\{\int_{B_{R}}[1+g(x,|D u|)] \mathrm{d} x\right\}^{1+\sigma} .
$$

Actually we prove the theorem under weak assumptions on $g$, (see $\left(H_{1}-H_{5}\right)$ and Th. 2.1 of Sect. 2).

The most relevant fact is that the integrand $f(x, \xi)$ may have exponential growth with respect to $\xi$, which involves non uniformily elliptic systems. Our result includes energy densities with variable growth as

$$
\int_{\Omega} a(x)[h(|D u|)]^{p(x)} \mathrm{d} x
$$

where $h$ is a $C^{2}([0,+\infty))$ positive convex function satisfying conditions (1.3) and (1.4) with $a, p \in W_{\text {loc }}^{1, \infty}(\Omega)$, $a(x), p(x) \geq c>0$ a.e. $x \in \Omega$; in particular we can take $h(t) \sim \exp \left(t^{m}\right)$ for $t \rightarrow+\infty$ and $m>0$.

The interest in functionals (1.1) with general growth and non uniformily elliptic systems (1.2) is motivated by several models which arise from different problems in mathematical physics: for example, the exponential growth is present in combustion theory, see Mosely [20] and in reaction of gases, see Aris [1]. Recently, this kind of systems has been used by Rajagopal and Rǔžička [21,22] in their model for the behaviour of special viscous fluids with the ability to change their mechanical properties in dependence on an applied electric field, 
the so-called electrorheological fluids. In fact, in the model proposed by Rajagopal and Růžička, the interaction between the electric field and the fluid in motion is expressed in the coefficients of the system by a variable exponent.

The particular case $f(x, \xi)=|\xi|^{p(x)}$ has been studied in the scalar case by ZhiKov [24], Mascolo and Papi [17] and Chiadò Piat and Coscia [5] (see also Marcellini [13,14] and Dall'Aglio et al. [7]). In the vectorial case, the regularity result is due to Coscia and Mingione [6] (see also Acerbi and Mingione [3,4] for related results).

For functionals with integrand of the type $(1.5)$ Migliorini in $[18,19]$ proves everywhere regularity of local minimizers in the context of $(p, q)$-growth conditions.

We improve these results to more general cases, like (1.7) and even to energies of the form

$$
g(x, t)=\exp \left(t^{p(x)}\right) \text { as } t \rightarrow+\infty,
$$

by using different techniques. We do not control the stored energy $g(x, t)$ by means of power functions: indeed we use its particular structure and properties directly (see also Dall'Aglio and Mascolo [8] for $L^{\infty}$-regularity).

The paper is organized as follows. Section 2 contains the statement of the general regularity theorem and some applications. In Section 3 we consider functionals with controllable growth, i.e. uniformly elliptic systems, and we prove for the gradient of minimizers an a priori estimate independent of the constants which appear in the controllability assumptions. In Section 4, we carry out the estimate to the general case by means of an approximation argument. More precisely, we construct a sequence of functions which converges to $g$ such that the corresponding functionals have controllable growth. By applying the a priori estimate, a procedure of passage to the limit gives estimate (1.6) for the minimizer of the original functional.

\section{Statement of the Regularity theorem}

Consider the integral functional

$$
F(u)=\int_{\Omega} f(x, D u(x)) \mathrm{d} x,
$$

where $\Omega$ is an open subset of $\mathbf{R}^{n}(n \geq 2), D u$ is the gradient of a vector-valued function $u: \Omega \rightarrow \mathbf{R}^{N}$, thus $D u=\left(u_{x_{i}}^{\alpha}\right)$ for $i=1, \ldots, n$ and $\alpha=1, \ldots, N$ is a matrix in $\mathbf{R}^{n N}$, and $f=f(x, \xi): \Omega \times \mathbf{R}^{n N} \rightarrow \mathbf{R}$ is a Carathéodory integrand.

We consider the case in which the stored energy $f$ depends on the modulus of the matrix $D u$ and satisfies general growth conditions. More precisely, we assume that

$$
f(x, \xi)=g(x,|\xi|),
$$

where $g(x, t): \Omega \times[0,+\infty) \rightarrow[0,+\infty)$ satisfies the following assumptions:

$\left(\mathbf{H}_{\mathbf{1}}\right)$ for a.e. $x \in \Omega, g(x, \cdot)$ is a positive convex function of class $C^{2}([0,+\infty))$ with $\frac{g_{t}(x, t)}{t}$ positive (strictly for $t>0)$ and increasing with respect to $t$ for a.e. $x \in \Omega$.

Observe that, since $\frac{g_{t}(x, t)}{t}$ is increasing, then necessarily $g_{t}(x, 0)=0$ for a.e. $x \in \Omega$. Moreover, without loss of generality, by adding a measurable bounded function of $x$ to $g$, we can reduce to the case $g(x, 0)=0$ for a.e. $x \in \Omega$.

Clearly from $\left(H_{1}\right)$ it follows that

$$
\begin{aligned}
& 0 \leq g(x, t) \leq g_{t}(x, t) t, \\
& 0 \leq g_{t}(x, t) \leq g_{t t}(x, t) t,
\end{aligned}
$$

$\forall t>0$ and a.e. $x \in \Omega$. 
$\left(\mathbf{H}_{2}\right)$ For every $\Omega_{0} \subset \subset \Omega$, there is a positive constant $\Lambda=\Lambda\left(\Omega_{0}\right)$ such that

$$
g_{t t}(x, t) \leq \Lambda, \quad \forall t \in[0,1] \text { and a.e. } x \in \Omega_{0},
$$

and a $t_{0} \in(0,1)$ and $\lambda=\lambda\left(\Omega_{0}\right)>0$ such that

$$
g\left(x, t_{0}\right) \geq \lambda, \quad \text { a.e. } x \in \Omega_{0} .
$$

The non oscillatory behaviour is included in the following assumption:

$\left(\mathbf{H}_{3}\right)$ For every $\Omega_{0} \subset \subset \Omega$ and $\alpha>1$, there exists a positive constant $c_{1}=c_{1}\left(\alpha, \Omega_{0}\right)$ such that

$$
g_{t t}(x, t) t^{2 \alpha} \leq c_{1}[g(x, t)]^{\alpha}, \quad \forall t \geq 1 \text { and a.e. } x \in \Omega_{0} .
$$

$\left(\mathbf{H}_{4}\right)$ For every $t \in[0,+\infty), g_{t}(x, t)$ admits weak derivatives $g_{t x_{s}}(x, t),(\forall s=1, \ldots, n)$, which are Carathéodory functions in $\Omega \times[0,+\infty)$ and locally integrable in $\Omega$. Moreover, for every $\Omega_{0} \subset \subset \Omega$ and $\alpha>1$ there exists a positive constant $c_{2}=c_{2}\left(\alpha, \Omega_{0}\right)$ such that

$$
\left|g_{t x_{s}}(x, t)\right| \leq c_{2} g_{t}(x, t)\left[1+g_{t}^{\alpha-1}(x, t)\right], \quad \forall t \geq 0 \text { and a.e. } x \in \Omega_{0} \text {. }
$$

$\left(\mathbf{H}_{\mathbf{5}}\right)$ For every $\Omega_{0} \subset \subset \Omega$ and $Q_{0}$ compact subset of $[1,+\infty), g_{t t}(x, t) \in L^{\infty}\left(\Omega_{0} \times Q_{0}\right)$.

By using (2.2) and (2.4), the following inequality holds (see [14,15] for details):

$$
\frac{g_{t}(x,|\xi|)}{|\xi|}|\lambda|^{2} \leq \sum_{i, j, \alpha, \beta} f_{\xi_{i}^{\alpha} \xi_{j}^{\beta}}(x, \xi) \lambda_{i}^{\alpha} \lambda_{j}^{\beta} \leq g_{t t}(x,|\xi|)|\lambda|^{2}
$$

for a.e. $x \in \Omega, \forall \xi, \lambda \in \mathbf{R}^{n N}$.

In the sequel, fixed $\Omega_{0} \subset \subset \Omega$ and $x_{0} \in \Omega_{0}$, we denote by $B_{\rho}$ and $B_{R}$ balls with the same center $x_{0}$ of radii $\rho$ and $R$ respectively compactly contained in $\Omega_{0},\left(0<\rho \leq R<\min \left\{\operatorname{dist}\left(x_{0}, \partial \Omega_{0}\right), 1\right\}\right)$.

Now we give the precise statement of our result.

Theorem 2.1. Consider the functional $F$ in (2.1) with $f(x, \xi)=g(x,|\xi|)$, where $g$ satisfies $\left(H_{1}-H_{5}\right)$. If $u$ is a local minimizer of $F$, then $u$ is of class $W_{\mathrm{loc}}^{1, \infty}\left(\Omega, \mathbf{R}^{N}\right)$ and there exists $\sigma=\sigma(n)>0$ such that

$$
\sup _{B_{\rho}}|D u| \leq c\left\{\int_{B_{R}}[1+g(x,|D u|)] \mathrm{d} x\right\}^{1+\sigma}
$$

where $c=c\left(n, N, c_{1}, c_{2}, \Lambda, \lambda, R, \rho\right)$.

Let now $h \in C^{2}([0,+\infty))$ be a strictly increasing convex function satisfying (1.3) and (1.4). Let $a(x), p(x) \in$ $W_{\mathrm{loc}}^{1, \infty}(\Omega)$ with $a(x), p(x) \geq c>0$ for a.e. $x \in \Omega$. The function

$$
g(x,|\xi|)=a(x) h(|\xi|)^{p(x)}
$$

with $h, a$ and $p$ such that $g(x, t)$ is of class $C^{2}$ with respect to $t$, models in natural way the assumptions $\left(H_{1}-H_{5}\right)$. It is easy to check that in (2.5) $\Lambda$ depends on $h^{\prime \prime}(1)$ and on an upper bound for $a(x)$ and $p(x)$, while in (2.8) $c_{2}=\max _{x \in \Omega_{0}}\left[\left|a_{x}(x) p(x)\right|+\left|a(x) p_{x}(x)\right|\right]$ where $\left|a_{x}(x)\right|$ and $\left|p_{x}(x)\right|$ denote the modulus of the gradient vectors of $a$ and $p$.

We observe explicitly that if $h(t)=t^{m}$ or $h(t)=t^{m} \ln (t+1)$ all the assumptions are satisfied provided $m p(x) \geq 2$ for a.e. $x \in \Omega$. 
On the other hand, if we consider exponential growth as

$$
\begin{aligned}
h(t) & \sim \exp \left(t^{m}\right) \quad \text { as } t \rightarrow+\infty, \text { with } m>0, \\
h(t) & \sim t^{\ln t \quad \text { as } t \rightarrow+\infty,} \\
h(t) & =\exp \left(t^{m}\right) \quad \text { with } m \geq 2,
\end{aligned}
$$

the variable exponent can be choosen such that $p(x) \geq \delta>0$ for a.e. $x \in \Omega$.

Moreover,

$$
g(x, t)=\exp \left(t^{p(x)}\right)
$$

as $t \rightarrow+\infty$ or even every other finite composition of exponentials as for example

$$
g(x, t)=\exp \left(\exp \left(t^{p_{1}(x)}\right)\right)^{p_{2}(x)},
$$

with $p_{i}(x) \geq 2,(i=1,2)$, satisfies $\left(H_{1}-H_{5}\right)$.

\section{A PRIORI ESTIMATES}

Marcellini in [15] proves some interesting inequalities in the case $g(x, t)=g(t)$ where $g$ is a positive, convex function of class $C^{2}$ satisfying (1.3) and the non oscillatory condition (1.4). Using assumptions $\left(H_{1}, H_{2}\right)$ and $\left(H_{3}\right)$, we can prove the same kind of inequalities for a.e. $x \in \Omega_{0} \subset \subset \Omega$. Moreover, it is easy to check that the uniform boundedness assumptions in $\left(H_{2}\right)$ imply that the constants in the pointwise inequalities are actually independent of $x \in \Omega_{0}$. These properties are contained in the following lemma (see Lems. 2.4, 2.6 and 2.7 of [15] for the proofs).

Lemma 3.1. Let $\Omega_{0} \subset \subset \Omega$ and $g$ satisfy $\left(H_{1}-H_{3}\right)$.

(i) For every $\alpha>1$ there exists a constant $c=c\left(\alpha, \Omega_{0}\right)$ such that

$$
\begin{aligned}
& g_{t}(x, t) t^{2 \alpha-1} \leq c[g(x, t)]^{\alpha}, \quad g_{t t}(x, t) t^{\alpha} \leq c\left[g_{t}(x, t)\right]^{\alpha} \\
& \forall t \geq 1, \quad \text { a.e. } x \in \Omega_{0} .
\end{aligned}
$$

(ii) For every $\alpha>1$ there exists a constant $c=c\left(\alpha, \Omega_{0}\right)$ such that

$$
\begin{aligned}
& 1+g_{t t}(x, t) t^{2 \alpha} \leq c[1+g(x, t)]^{\alpha}, \\
& \forall t \geq 0, \quad \text { a.e. } x \in \Omega_{0} .
\end{aligned}
$$

(iii) For every $\beta>2$ there exists a constant $c=c\left(\beta, \Omega_{0}\right)$ such that $\forall \gamma \geq 0$

$$
\begin{aligned}
& 1+g_{t t}(x, t)\left(\frac{t^{\gamma+1}}{\gamma+1}\right)^{\beta} \leq c\left[1+\int_{0}^{t} s^{\gamma} \sqrt{\frac{g_{t}(x, s)}{s}} \mathrm{~d} s\right]^{\beta}, \\
& \forall t \geq 0, \quad \text { a.e. } x \in \Omega_{0} .
\end{aligned}
$$

The constants in (i-iii) depend on $\Lambda$ and $\lambda$ in $\left(H_{2}\right)$.

We make the following supplementary assumptions (which will be removed through the approximation method in Sect. 4).

Assume that there exist positive constants $m, M$ and $N$, depending on $\Omega_{0} \subset \subset \Omega$, such that

$$
m \leq \frac{g_{t}(x, t)}{t} \leq g_{t t}(x, t) \leq M
$$


and

$$
\left|g_{t x_{s}}(x, t)\right| \leq N\left(1+t^{2}\right)^{\frac{1}{2}}
$$

$\forall t>0$ and for a.e. $x \in \Omega_{0}$. By taking in account $(2.9,3.1)$ implies the uniform ellipticity condition, i.e.

$$
m|\lambda|^{2} \leq \sum_{i, j, \alpha, \beta} f_{\xi_{i}^{\alpha} \xi_{j}^{\beta}}(x, \xi) \lambda_{i}^{\alpha} \lambda_{j}^{\beta} \leq M|\lambda|^{2},
$$

and, since

equation (3.2) gives

$$
\left|f_{\xi_{i}^{\alpha} x_{s}}(x, \xi)\right| \leq\left|g_{t x_{s}}(x,|\xi|)\right|, \quad \text { a.e. } x \in \Omega_{0}, \quad \forall \xi \in \mathbf{R}^{n N}
$$

$$
\left|f_{\xi_{i}^{\alpha} x_{s}}(x, \xi)\right| \leq N\left(1+|\xi|^{2}\right)^{\frac{1}{2}}, \quad \text { a.e. } x \in \Omega_{0}, \quad \forall \xi \in \mathbf{R}^{n N} .
$$

First we present the following intermediate regularity result:

Proposition 3.2. Consider the functional $F$ in (2.1) with $f(x, \xi)=g(x,|\xi|)$ where $g$ satisfies $\left(H_{1}-H_{4}\right)$ and (3.1, 3.2) and let $u$ be a local minimizer of $F$. Then $u \in W_{\mathrm{loc}}^{1, \infty}\left(\Omega, \mathbf{R}^{N}\right)$ and, for every $\Omega_{0} \subset \subset \Omega$ and $0<\rho<R<1$ such that $B_{R} \subset \subset \Omega_{0}$, there exists $\sigma=\sigma(n)>0$ such that the following estimate holds

$$
\sup _{B_{\rho}}|D u| \leq c\left\{\int_{B_{R}}[1+g(x,|D u|)] \mathrm{d} x\right\}^{1+\sigma}
$$

where $c$ depends on $n, N, R, \rho$ and on the constants in $\left(H_{1}-H_{4}\right)$.

The proof follows by collecting Lemmas 3.3 and 3.4 below.

In the sequel, we denote by $1^{*}=\frac{n}{n-1}$ and by $2^{*}=\frac{2 n}{n-2}$ if $n>2$, while $2^{*}$ is any real number strictly greater than $1^{*} 2$, when $n=2$.

Lemma 3.3. Let $\left(H_{1}-H_{4}\right)$ and (3.1, 3.2) hold. If $u$ is a local minimizer of $F$ in (2.1), then $u \in W_{\text {loc }}^{1, \infty}\left(\Omega, \mathbf{R}^{N}\right)$ and there exists $c>0$, depending on $n, N$ and on the constants in $\left(H_{1}-H_{4}\right)$, such that the following estimate holds

$$
\sup _{B_{\rho}}|D u| \leq \frac{c}{(R-\rho)^{n-1}}\left\{\int_{B_{R}}\left[1+|D u|^{*^{*} 2} g_{t t}(x,|D u|)\right]^{\frac{2^{*}}{1^{*}}} \mathrm{~d} x\right\}^{\frac{1}{1 *}} .
$$

Proof. Let $u$ be a local minimizer of (2.1). By the left hand side of (3.3), $u$ satisfies the Euler's first variation:

$$
\int_{\Omega} \sum_{i, \alpha} f_{\xi_{i}^{\alpha}}(x, D u) \varphi_{x_{i}}^{\alpha}(x) \mathrm{d} x=0, \quad \forall \varphi=\left(\varphi^{\alpha}\right) \in W_{0}^{1,2}\left(\Omega, \mathbf{R}^{N}\right) .
$$

The technique of the difference quotient (see $[10,12]$ or in the context of non standard growth $[15,19]$ ) gives that $u$ admits second derivatives, precisely $u \in W_{\text {loc }}^{2,2}\left(\Omega, \mathbf{R}^{N}\right)$ and satisfies the second variation

$$
\begin{gathered}
\int_{\Omega}\left\{\sum_{i, j, \alpha, \beta} f_{\xi_{i}^{\alpha} \xi_{j}^{\beta}}(x, D u) \varphi_{x_{i}}^{\alpha} u_{x_{s} x_{j}}^{\beta}+\sum_{i, \alpha} f_{\xi_{i}^{\alpha} x_{s}}(x, D u) \varphi_{x_{i}}^{\alpha}\right\} \mathrm{d} x=0, \\
\forall s=1, \ldots, n, \quad \forall \varphi=\left(\varphi^{\alpha}\right) \in W_{0}^{1,2}\left(\Omega, \mathbf{R}^{N}\right) .
\end{gathered}
$$

Let $\Omega_{0} \subset \subset \Omega$ and $\eta$ be a positive function of class $C_{0}^{1}\left(\Omega_{0}\right)$; fixed $s \in\{1, \ldots, n\}$, we choose $\varphi^{\alpha}=\eta^{2} u_{x_{s}}^{\alpha} \Phi(|D u|)$ for every $\alpha=1, \ldots, N$, where $\Phi$ is a positive, increasing, bounded, Lipschitz continuous function defined in $[0,+\infty)$ (in particular $\Phi$ and $\Phi^{\prime}$ are bounded, so that $\left.\varphi=\left(\varphi^{\alpha}\right) \in W_{0}^{1,2}\left(\Omega, \mathbf{R}^{N}\right)\right)$. Then

$$
\varphi_{x_{i}}^{\alpha}=2 \eta \eta_{x_{i}} u_{x_{s}}^{\alpha} \Phi(|D u|)+\eta^{2} u_{x_{s} x_{i}}^{\alpha} \Phi(|D u|)+\eta^{2} u_{x_{s}}^{\alpha} \Phi^{\prime}(|D u|)(|D u|)_{x_{i}}
$$


and from (3.6) we obtain

$$
\begin{aligned}
0= & \int_{\Omega} \eta^{2} \Phi \sum_{i, j, \alpha, \beta} f_{\xi_{i}^{\alpha} \xi_{j}^{\beta}}(x, D u) u_{x_{s} x_{i}}^{\alpha} u_{x_{s} x_{j}}^{\beta} \mathrm{d} x+\int_{\Omega} 2 \eta \Phi \sum_{i, j, \alpha, \beta} f_{\xi_{i}^{\alpha} \xi_{j}^{\beta}}(x, D u) \eta_{x_{i}} u_{x_{s}}^{\alpha} u_{x_{s} x_{j}}^{\beta} \mathrm{d} x \\
& +\int_{\Omega} \eta^{2} \Phi^{\prime} \sum_{i, j, \alpha, \beta} f_{\xi_{i}^{\alpha} \xi_{j}^{\beta}}(x, D u) u_{x_{s}}^{\alpha} u_{x_{s} x_{j}}^{\beta}(|D u|)_{x_{i}} \mathrm{~d} x \\
& +\int_{\Omega} 2 \eta \Phi \sum_{i, \alpha} f_{\xi_{i}^{\alpha} x_{s}}(x, D u) \eta_{x_{i}} u_{x_{s}}^{\alpha} \mathrm{d} x \\
& +\int_{\Omega} \eta^{2} \Phi \sum_{i, \alpha} f_{\xi_{i}^{\alpha} x_{s}}(x, D u) u_{x_{s} x_{i}}^{\alpha} \mathrm{d} x \\
& +\int_{\Omega} \eta^{2} \Phi^{\prime} \sum_{i, \alpha} f_{\xi_{i}^{\alpha} x_{s}}(x, D u) u_{x_{s}}^{\alpha}(|D u|)_{x_{i}} \mathrm{~d} x \\
= & I_{1}+I_{2}+I_{3}+I_{4}+I_{5}+I_{6}
\end{aligned}
$$

(here and in the following we write only $\Phi$ and $\Phi^{\prime}$ instead of $\Phi(|D u|)$ and $\Phi^{\prime}(|D u|)$ ). We sum with respect to $s$ from 1 to $n$ the previous equation but we still indicate the integrals with $I_{1}-I_{6}$. In the sequel we denote by $c$ any constant which may take different values from line to line and depends on the constants in assumptions $\left(H_{1}-H_{4}\right)$ and on the dimensions $n$ and $N$.

Let us start with the estimate of the integral $I_{2}$. By Cauchy-Schwartz inequality, Young's inequality $a b \leq$ $\epsilon a^{2}+\frac{b^{2}}{4 \epsilon}, \forall \epsilon>0$, and $(2.9)$

$$
\begin{aligned}
\left|I_{2}\right| & =\left|\int_{\Omega} 2 \eta \Phi \sum_{i, j, s, \alpha, \beta} f_{\xi_{i}^{\alpha} \xi_{j}^{\beta}}(x, D u) \eta_{x_{i}} u_{x_{s}}^{\alpha} u_{x_{s} x_{j}}^{\beta} \mathrm{d} x\right| \\
& \leq \int_{\Omega} 2 \eta \Phi\left\{\sum_{i, j, s, \alpha, \beta} f_{\xi_{i}^{\alpha} \xi_{j}^{\beta}}(x, D u) \eta_{x_{i}} u_{x_{s}}^{\alpha} \eta_{x_{j}} u_{x_{s}}^{\beta}\right\}^{\frac{1}{2}}\left\{\sum_{i, j, s, \alpha, \beta} f_{\xi_{i}^{\alpha} \xi_{j}^{\beta}}(x, D u) u_{x_{s} x_{i}}^{\alpha} u_{x_{s} x_{j}}^{\beta}\right\}^{\frac{1}{2}} \mathrm{~d} x \\
& \leq c \epsilon_{1} \int_{\Omega} \eta^{2} \Phi \sum_{i, j, s, \alpha, \beta} f_{\xi_{i}^{\alpha} \xi_{j}^{\beta}}(x, D u) u_{x_{s} x_{i}}^{\alpha} u_{x_{s} x_{j}}^{\beta} \mathrm{d} x+\frac{c}{4 \epsilon_{1}} \int_{\Omega}|D \eta|^{2} \Phi g_{t t}(x,|D u|)|D u|^{2} \mathrm{~d} x .
\end{aligned}
$$

Let us consider $I_{3}$. Since $f(x, \xi)=g(x,|\xi|)$, we have

$$
\begin{aligned}
f_{\xi_{i}^{\alpha}}(x, \xi) & =\frac{g_{t}(x,|\xi|)}{|\xi|} \xi_{i}^{\alpha} \\
f_{\xi_{i}^{\alpha} \xi_{j}^{\beta}}(x, \xi) & =\left(\frac{g_{t t}(x,|\xi|)}{|\xi|^{2}}-\frac{g_{t}(x,|\xi|)}{|\xi|^{3}}\right) \xi_{j}^{\beta} \xi_{i}^{\alpha}+\frac{g_{t}(x,|\xi|)}{|\xi|} \delta_{\xi_{i}^{\alpha}} \xi_{j}^{\beta} .
\end{aligned}
$$

Using (2.4) and the fact that $g_{t}(x, t)$ is positive, we can prove that

$$
\sum_{i, j, s, \alpha, \beta} f_{\xi_{i}^{\alpha} \xi_{j}^{\beta}}(x, D u) u_{x_{s}}^{\alpha} u_{x_{s} x_{j}}^{\beta}(|D u|)_{x_{i}} \geq 0 .
$$


In fact

$$
\begin{aligned}
\sum_{i, j, s, \alpha, \beta} f_{\xi_{i}^{\alpha} \xi_{j}^{\beta}}(x, D u) u_{x_{s}}^{\alpha} u_{x_{s} x_{j}}^{\beta}(|D u|)_{x_{i}}= & \left(\frac{g_{t t}(x,|D u|)}{|D u|}-\frac{g_{t}(x,|D u|)}{|D u|^{2}}\right) \sum_{i, s, \alpha}\left[u_{x_{i}}^{\alpha}(|D u|)_{x_{s}}\right]^{2} \\
& +g_{t}(x,|D u|) \sum_{i}(|D u|)_{x_{i}}^{2} \geq 0
\end{aligned}
$$

since $(|D u|)_{x_{i}}=\frac{1}{D u} \sum_{s, \alpha} u_{x_{s}}^{\alpha} u_{x_{i} x_{s}}^{\alpha}$; hence (3.9) is proved and this easily implies that $I_{3} \geq 0$. Consider now $I_{4}$ : by assumption $\left(H_{4}\right)$ and by (3.4) and (2.4), we have

$$
\begin{aligned}
\left|I_{4}\right| & =\left|\int_{\Omega} 2 \eta \Phi \sum_{i, s, \alpha} f_{\xi_{i}^{\alpha} x_{s}}(x, D u) \eta_{x_{i}} u_{x_{s}}^{\alpha} \mathrm{d} x\right| \\
& \leq c \int_{\Omega} 2 \eta \Phi g_{t t}(x,|D u|)|D u|\left[1+g_{t}^{\alpha-1}(x,|D u|)\right] \sum_{i, s, \alpha}\left|\eta_{x_{i}} u_{x_{s}}^{\alpha}\right| \mathrm{d} x \\
& \leq c \int_{\Omega} 2 \eta|D \eta| \Phi g_{t t}(x,|D u|)|D u|^{2}\left[1+g_{t}^{\alpha-1}(x,|D u|)\right] \mathrm{d} x .
\end{aligned}
$$

In order to estimate $I_{5}$, let us observe that, taking in account $(2.4,2.8)$ becomes

$$
\left|g_{t x_{s}}(x, t)\right| \leq c_{2}\left\{\frac{g_{t}(x, t)}{t}\right\}^{\frac{1}{2}}\left\{g_{t t}(x, t) t^{2}\left[1+g_{t}^{2(\alpha-1)}(x, t)\right]\right\}^{\frac{1}{2}}
$$

thus, by using Cauchy-Schwartz inequality and Young's inequality, we obtain

$$
\begin{aligned}
\left|I_{5}\right|= & \left|\int_{\Omega} \eta^{2} \Phi \sum_{i, s, \alpha} f_{\xi_{i}^{\alpha} x_{s}}(x, D u) u_{x_{s} x_{i}}^{\alpha} \mathrm{d} x\right| \leq \int_{\Omega} \eta^{2} \Phi\left\{\sum_{i, s, \alpha} f_{\xi_{i}^{\alpha} x_{s}}^{2}(x, D u)\right\}^{\frac{1}{2}}\left|D^{2} u\right| \mathrm{d} x \\
\leq & c_{2} \int_{\Omega} \eta^{2} \Phi\left\{\frac{g_{t}(x,|D u|)}{|D u|}\left|D^{2} u\right|^{2}\right\}^{\frac{1}{2}}\left\{g_{t t}(x,|D u|)|D u|^{2}\left[1+g_{t}^{2(\alpha-1)}(x, t)\right]\right\}^{\frac{1}{2}} \mathrm{~d} x \\
\leq & c \epsilon_{2} \int_{\Omega} \eta^{2} \Phi \frac{g_{t}(x,|D u|)}{|D u|}\left|D^{2} u\right|^{2} \mathrm{~d} x \\
& +\frac{c}{4 \epsilon_{2}} \int_{\Omega} \eta^{2} \Phi g_{t t}(x,|D u|)|D u|^{2}\left[1+g_{t}^{2(\alpha-1)}(x,|D u|)\right] \mathrm{d} x .
\end{aligned}
$$

Similarly

$$
\begin{aligned}
\left|I_{6}\right|= & \left|\int_{\Omega} \eta^{2} \Phi^{\prime} \sum_{i, s, \alpha} f_{\xi_{i}^{\alpha} x_{s}}(x, D u) u_{x_{s}}^{\alpha}(|D u|)_{x_{i}} \mathrm{~d} x\right| \leq c_{1} \int_{\Omega} \eta^{2} \Phi^{\prime}|D u|\left\{\frac{g_{t}(x,|D u|)}{|D u|} \sum_{i}(|D u|)_{x_{i}}^{2}\right\}^{\frac{1}{2}} \mathrm{~d} x \\
& \times\left\{g_{t t}(x,|D u|)|D u|^{2}\left[1+g_{t}^{2(\alpha-1)}(x,|D u|)\right]\right\}^{\frac{1}{2}} \mathrm{~d} x \\
\leq & c \epsilon_{3} \int_{\Omega} \eta^{2} \Phi^{\prime}|D u| \frac{g_{t}(x,|D u|)}{|D u|} \sum_{i}(|D u|)_{x_{i}}^{2} \mathrm{~d} x \\
& +\frac{c}{4 \epsilon_{3}} \int_{\Omega} \eta^{2} \Phi^{\prime}|D u| g_{t t}(x,|D u|)|D u|^{2}\left[1+g_{t}^{2(\alpha-1)}(x,|D u|)\right] \mathrm{d} x
\end{aligned}
$$


Collecting (3.8-3.13) and choosing $\epsilon_{1}$ sufficiently small we have also

$$
\begin{aligned}
\int_{\Omega} \eta^{2} \Phi \sum_{i, j, s, \alpha, \beta} f_{\xi_{i}^{\alpha} \xi_{j}^{\beta}}(x, D u) u_{x_{s} x_{i}}^{\alpha} u_{x_{s} x_{j}}^{\beta} \mathrm{d} x \leq & c \int_{\Omega}|D \eta|^{2} \Phi g_{t t}(x,|D u|)|D u|^{2} \mathrm{~d} x \\
& +c \int_{\Omega} 2 \eta|D \eta| \Phi g_{t t}(x,|D u|)|D u|^{2}\left[1+g_{t}^{\alpha-1}(x,|D u|)\right] \mathrm{d} x \\
& +c \epsilon_{2} \int_{\Omega} \eta^{2} \Phi \frac{g_{t}(x,|D u|)}{|D u|}\left|D^{2} u\right|^{2} \mathrm{~d} x \\
& +\frac{c}{4 \epsilon_{2}} \int_{\Omega} \eta^{2} \Phi g_{t t}(x,|D u|)|D u|^{2}\left[1+g_{t}^{2(\alpha-1)}(x,|D u|)\right] \mathrm{d} x \\
& +c \epsilon_{3} \int_{\Omega} \eta^{2} \Phi^{\prime}|D u| \frac{g_{t}(x,|D u|)}{|D u|} \sum_{i}(|D u|)_{x_{i}}^{2} \mathrm{~d} x \\
& +\frac{c}{4 \epsilon_{3}} \int_{\Omega} \eta^{2} \Phi^{\prime}|D u| g_{t t}(x,|D u|)|D u|^{2}\left[1+g_{t}^{2(\alpha-1)}(x,|D u|)\right] \mathrm{d} x .
\end{aligned}
$$

By choosing $\epsilon_{2}$ sufficiently small, the left inequality of (2.9), implies

$$
\begin{aligned}
\int_{\Omega} \eta^{2} \Phi \frac{g_{t}(x,|D u|)}{|D u|}\left|D^{2} u\right|^{2} \mathrm{~d} x \leq & c \int_{\Omega}|D \eta|^{2} \Phi g_{t t}(x,|D u|)|D u|^{2} \mathrm{~d} x \\
& +c \int_{\Omega} 2 \eta|D \eta| \Phi g_{t t}(x,|D u|)|D u|^{2}\left[1+g_{t}^{\alpha-1}(x,|D u|)\right] \mathrm{d} x \\
& +c \int_{\Omega} \eta^{2} \Phi g_{t t}(x,|D u|)|D u|^{2}\left[1+g_{t}^{2(\alpha-1)}(x,|D u|)\right] \mathrm{d} x \\
& +c \epsilon_{3} \int_{\Omega} \eta^{2} \Phi^{\prime}|D u| \frac{g_{t}(x,|D u|)}{|D u|} \sum_{i}(|D u|)_{x_{i}}^{2} \mathrm{~d} x \\
& +\frac{c}{4 \epsilon_{3}} \int_{\Omega} \eta^{2} \Phi^{\prime}|D u| g_{t t}(x,|D u|)|D u|^{2}\left[1+g_{t}^{2(\alpha-1)}(x,|D u|)\right] \mathrm{d} x .
\end{aligned}
$$

Now we allow only test function $\Phi$ satisfying

$$
\Phi^{\prime}(t) t \leq c_{\Phi} \Phi(t)
$$

for a certain constant $c_{\Phi} \geq 0$. Recalling that $(|D u|)_{x_{i}}=\frac{1}{|D u|} \sum_{s, \alpha} u_{x_{s}}^{\alpha} u_{x_{i} x_{s}}^{\alpha}$, and using Cauchy-Schwartz inequality, we see that

$$
|D(|D u|)|^{2}=\sum_{i}(|D u|)_{x_{i}}^{2} \leq \sum_{i, s, \alpha}\left|u_{x_{s} x_{i}}^{\alpha}\right|^{2}=\left|D^{2} u\right|^{2}
$$

We use the last inequality to estimate the first member in (3.15) and for small $\epsilon_{3}$ we get

$$
\begin{aligned}
\int_{\Omega} \eta^{2} \Phi \frac{g_{t}(x,|D u|)}{|D u|} \sum_{i}(|D u|)_{x_{i}}^{2} \mathrm{~d} x \leq & c \int_{\Omega}|D \eta|^{2} \Phi g_{t t}(x,|D u|)|D u|^{2} \mathrm{~d} x \\
& +c \int_{\Omega} 2 \eta|D \eta| \Phi g_{t t}(x,|D u|)|D u|^{2}\left[1+g_{t}^{\alpha-1}(x,|D u|)\right] \mathrm{d} x \\
& +c \int_{\Omega} \eta^{2} \Phi g_{t t}(x,|D u|)|D u|^{2}\left[1+g_{t}^{2(\alpha-1)}(x,|D u|)\right] \mathrm{d} x \\
& +c\left(c_{\Phi}\right)^{2} \int_{\Omega} \eta^{2} \Phi g_{t t}(x,|D u|)|D u|^{2}\left[1+g_{t}^{2(\alpha-1)}(x,|D u|)\right] \mathrm{d} x
\end{aligned}
$$


On the other hand, since $2 \eta|D \eta|,|D \eta|^{2}, \eta^{2}$ are less then or equal to $\eta^{2}+|D \eta|^{2}$, using (3.17) we finally have

$$
\int_{\Omega} \eta^{2} \Phi \frac{g_{t}(x,|D u|)}{|D u|}|D(|D u|)|^{2} \mathrm{~d} x \leq c\left(1+c_{\Phi}\right)^{2} \int_{\Omega}\left[\eta^{2}+|D \eta|^{2}\right] \Phi g_{t t}(x,|D u|)|D u|^{2}\left[1+g_{t}^{2(\alpha-1)}(x,|D u|)\right] \mathrm{d} x,
$$

where $c=c\left(n, N, \Omega_{0}, \Lambda, \lambda, c_{1}, c_{2}\right)$. Let now $\Phi$ be a positive, increasing and locally Lipschitz continuous function in $[0,+\infty)$ satisfying $(3.16)$. Then we can approximate $\Phi$ by a sequence of Lipschitz functions $\Phi_{r}$ bounded with $\Phi_{r}^{\prime}$ bounded, in the following way:

$$
\Phi_{r}(t)=\left\{\begin{array}{ll}
\Phi(t) & \text { for } t \in[0, r] \\
\Phi(r) & \text { for } t \in(r,+\infty)
\end{array} \quad r \in \mathbb{N} .\right.
$$

Since $\Phi_{r}^{\prime}(t) t \leq c_{\Phi} \Phi(t)$, while $\Phi_{r}^{\prime}\left(r^{+}\right)$and $\Phi_{r}^{\prime}\left(r^{-}\right)$are uniformly bounded, the condition (3.16) holds for $\Phi_{r}$ with the same constant $c_{\Phi}$, thus (3.19) holds $\Phi_{r}$. By monotone convergence theorem, letting $r$ tend to $+\infty$, we infer that (3.19) holds for such a $\Phi$.

For $t \in[0,+\infty)$ and $x \in \Omega$ define

$$
G(x, t)=1+\int_{0}^{t} \sqrt{\Phi(s) \frac{g_{t}(x, s)}{s}} \mathrm{~d} s
$$

since the integrand function is increasing and by $(2.4)$, we get

$$
[G(x, t)]^{2} \leq\left[1+t \sqrt{\Phi(t) \frac{g_{t}(x, t)}{t}}\right]^{2} \leq 2\left[1+t^{2} \Phi(t) \frac{g_{t}(x, t)}{t}\right] \leq 2\left[1+\Phi(t) g_{t t}(x, t) t^{2}\right] .
$$

Moreover, by $\left(H_{4}\right), \forall i=1, \ldots, n$ we have

$$
\begin{aligned}
{\left[\frac{\partial}{\partial x_{i}} G(x, t)\right]^{2}=\left[\int_{0}^{t} \sqrt{\frac{\Phi(s)}{s}} \frac{g_{t x_{i}}(x, s)}{2 \sqrt{g_{t}(x, s)}} \mathrm{d} s\right]^{2} } & \leq c\left[t \sqrt{\frac{\Phi(t)}{t} g_{t}(x, t)}\left[1+g_{t}^{\alpha-1}(x, t)\right]\right]^{2} \\
& \leq c \Phi(t) g_{t t}(x, t) t^{2}\left[1+g_{t}^{2(\alpha-1)}(x, t)\right]
\end{aligned}
$$

We denote by $D_{x} G$ the weak gradient of $G(x, t)$ with respect to $x$. The assumptions $\left(H_{1}\right)$ and $\left(H_{4}\right)$ ensure (see for instance Marcus and Mizel [16]) that the chain rule holds and the previous estimates yield:

$$
\begin{aligned}
|D[\eta G(x,|D u|)]|^{2} \leq & c|D \eta|^{2}[G(x,|D u|)]^{2}+c \eta^{2}\left[G_{t}(x,|D u|) D(|D u|)\right]^{2}+c \eta^{2}\left[D_{x} G(x,|D u|)\right]^{2} \\
\leq & c|D \eta|^{2}\left[1+\Phi g_{t t}(x,|D u|)|D u|^{2}\right]+c \eta^{2} \Phi \frac{g_{t}(x,|D u|)}{|D u|}|D(|D u|)|^{2} \\
& +c \eta^{2} \Phi g_{t t}(x,|D u|)|D u|^{2}\left[1+g_{t}^{2(\alpha-1)}(x,|D u|)\right] .
\end{aligned}
$$

Therefore by (3.19), we deduce

$$
\int_{\Omega}|D[\eta G(x,|D u|)]|^{2} \mathrm{~d} x \leq c\left(1+c_{\Phi}\right)^{2} \int_{\Omega}\left[\eta^{2}+|D \eta|^{2}\right] \Phi\left[1+g_{t t}(x,|D u|)|D u|^{2}\right]\left[1+g_{t}^{2(\alpha-1)}(x,|D u|)\right] \mathrm{d} x
$$


where $c=c\left(n, N, \Omega_{0}, \Lambda, \lambda, c_{1}, c_{2}, \alpha\right)$. Let $2^{*}=\frac{2 n}{n-2}$ for $n>2$, while $2^{*}$ equal to any fixed real number greater than $1^{*} 2$ if $n=2$. By Sobolev's inequality:

$$
\left\{\int_{\Omega} \eta^{2^{*}}[G(x,|D u|)]^{2^{*}} \mathrm{~d} x\right\}^{\frac{2}{2^{*}}} \leq c\left(1+c_{\Phi}\right)^{2} \int_{\Omega}\left[\eta^{2}+|D \eta|^{2}\right]\left[1+\Phi g_{t t}(x,|D u|)|D u|^{2}\right]\left[1+g_{t}^{2(\alpha-1)}(x,|D u|)\right] \mathrm{d} x .
$$

Choose $\Phi(t)=t^{2 \gamma}$ with $\gamma \geq 0$, thus the condition (3.16) is satisfied with $c_{\Phi}=2 \gamma$. With this choice of $\Phi$, equation (3.20) reduces to

$$
\left\{\int_{\Omega} \eta^{2^{*}}[G(x,|D u|)]^{2^{*}} \mathrm{~d} x\right\}^{\frac{2}{2^{*}}} \leq c(1+\gamma)^{2} \int_{\Omega}\left[\eta^{2}+|D \eta|^{2}\right]\left[1+|D u|^{2(\gamma+1)} g_{t t}(x,|D u|)\right]\left[1+g_{t}^{2(\alpha-1)}(x,|D u|)\right] \mathrm{d} x .
$$

By (iii) of Lemma 3.1, for a.e. $x \in \Omega_{0}$ we get

$$
[G(x, t)]^{2^{*}}=\left[1+\int_{0}^{t} s^{\gamma} \sqrt{\frac{g_{t}(x, s)}{s}} \mathrm{~d} s\right]^{2^{*}} \geq c\left[1+\left(\frac{t^{\gamma+1}}{\gamma+1}\right)^{2^{*}} g_{t t}(x, t)\right]
$$

thus (3.21) becomes

$$
\begin{aligned}
& \left\{\int_{\Omega} \eta^{2^{*}}\left[1+|D u|^{2^{*}(\gamma+1)} g_{t t}(x,|D u|)\right] \mathrm{d} x\right\}^{\frac{2}{2^{*}}} \\
& \quad \leq c(1+\gamma)^{4} \int_{\Omega}\left[\eta^{2}+|D \eta|^{2}\right]\left[1+|D u|^{2(\gamma+1)} g_{t t}(x,|D u|)\right]\left[1+g_{t}^{2(\alpha-1)}(x,|D u|)\right] \mathrm{d} x
\end{aligned}
$$

Fixed $\rho_{0}$ and $R_{0}$ such that $B_{\rho_{0}} \subset \subset B_{R_{0}} \subset \subset \Omega_{0}$, for $0<\rho_{0}<\rho<R<R_{0}$, let $\eta$ be a positive test function equal to 1 in $B_{\rho}$, whose support is contained in $B_{R}$, such that $|D \eta| \leq \frac{2}{R-\rho}$. Set $\theta=\gamma+1$ and $\epsilon=2(\alpha-1)$, using (2.4) we have

$$
\left\{\int_{B_{\rho}}\left[1+|D u|^{2^{*} \theta} g_{t t}(x,|D u|)\right] \mathrm{d} x\right\}^{\frac{2}{2^{*}}} \leq c \frac{\theta^{4}}{(R-\rho)^{2}} \int_{B_{R}}\left[1+|D u|^{2 \theta} g_{t t}(x,|D u|) g_{t t}^{\epsilon}(x,|D u|)|D u|^{\epsilon}\right] \mathrm{d} x .
$$

For an arbitrary $\tau, 0<\tau<1$, using Hölder inequality we get

$$
\begin{aligned}
\int_{B_{R}}\left[1+|D u|^{2 \theta} g_{t t}(x,|D u|) g_{t t}^{\epsilon}(x,|D u|)|D u|^{\epsilon}\right] \mathrm{d} x & \leq c \int_{B_{R}}\left[1+|D u|^{2 \theta} g_{t t}^{1-\tau}(x,|D u|) g_{t t}^{\epsilon+\tau}(x,|D u|)|D u|^{\epsilon}\right] \mathrm{d} x \\
\leq & c\left\{\int_{B_{R}}\left[1+|D u|^{\frac{2 \theta}{1-\tau}} g_{t t}(x,|D u|)\right] \mathrm{d} x\right\}^{1-\tau} \\
& \times\left\{\int_{B_{R}}\left[1+g_{t t^{\frac{\tau+\epsilon}{\tau}}}(x,|D u|)|D u|^{\frac{\epsilon}{\tau}}\right] \mathrm{d} x\right\}^{\tau} .
\end{aligned}
$$

Moreover, by $\left(H_{2}\right)$

$$
1+g_{t t}^{\frac{\tau+\epsilon}{\tau}}(x, t) t^{\frac{\epsilon}{\tau}} \leq c\left[1+g_{t t}(x, t) t^{\frac{\epsilon}{\tau+\epsilon}}\right]^{\frac{\tau+\epsilon}{\tau}} \leq c\left[1+g_{t t}(x, t) t^{t^{*} 2}\right]^{\frac{\tau+\epsilon}{\tau}}
$$


and then by (3.22)

$$
\begin{aligned}
\left\{\int_{B_{\rho}}\left[1+|D u|^{2^{*} \theta} g_{t t}(x,|D u|)\right] \mathrm{d} x\right\}^{\frac{2}{2^{*}}} \leq & c \frac{\theta^{4}}{(R-\rho)^{2}}\left\{\int_{B_{R}}\left[1+|D u|^{\frac{2 \theta}{1-\tau}} g_{t t}(x,|D u|)\right] \mathrm{d} x\right\}^{1-\tau} \\
& \times\left\{\int_{B_{R}}\left[1+g_{t t}(x,|D u|)|D u|^{1^{*} 2}\right]^{\frac{\tau+\epsilon}{\tau}} \mathrm{d} x\right\}^{\tau} .
\end{aligned}
$$

To apply an iteration procedure, we need $\frac{2}{1-\tau}<2^{*}$, then it is sufficient that $\tau<\frac{2}{n}$. Choose $\tau=\frac{1}{n}$, thus $\frac{2}{1-\tau}=1^{*} 2<2^{*}$ and let $\epsilon$ such that $\frac{\tau+\epsilon}{\tau}=1+\epsilon n=\frac{2^{*}}{1^{*} 2}$. Since $u \in W_{\mathrm{loc}}^{2,2}\left(\Omega, \mathbf{R}^{N}\right)$, then $D u \in L_{\mathrm{loc}}^{2^{*}}\left(\Omega, \mathbf{R}^{n N}\right)$ and recalling that $g_{t t}$ satisfies the supplementary assumption (3.1), we deduce that following integral is finite:

$$
\mathcal{A}=\int_{B_{R_{0}}}\left[1+g_{t t}(x,|D u|)|D u|^{*^{*} 2}\right]^{\frac{2^{*}}{1^{*}}} \mathrm{~d} x
$$

and (3.24) becomes

$$
\left\{\int_{B_{\rho}}\left[1+|D u|^{2^{*} \theta} g_{t t}(x,|D u|)\right] \mathrm{d} x\right\}^{\frac{1}{2^{*}}} \leq c \frac{\theta^{2}}{R-\rho}\left\{\int_{B_{R}}\left[1+|D u|^{*} 2 \theta g_{t t}(x,|D u|)\right] \mathrm{d} x\right\}^{\frac{1}{1^{* 2}}} \mathcal{A}^{\frac{1}{2 n}} .
$$

We define a sequence of exponents $\theta_{j}$ in the following way:

$$
\begin{aligned}
& \theta_{0}=1 \\
& \theta_{j}=\frac{2^{*}}{1^{*} 2} \theta_{j-1}, \quad \forall j=1,2, \ldots
\end{aligned}
$$

or equivalently $\theta_{0}=1$ and $\theta_{j}=\left(\frac{2^{*}}{1^{*} 2}\right)^{j}, \forall j=1,2, \ldots$

Define also $\rho_{j}=\rho_{0}+\frac{R_{0}-\rho_{0}}{2^{j}}$ for $j=0,1,2, \ldots$ and

$$
A_{j}=\left\{\int_{B_{\rho_{j}}}\left[1+|D u|^{1^{*} 2 \theta_{j}} g_{t t}(x,|D u|)\right] \mathrm{d} x\right\}^{\frac{1}{1^{*} 2 \theta_{j}}}
$$

and insert in (3.26) $R=\rho_{j}, \rho=\rho_{j+1}$ and $\theta=\theta_{j}$. Since $R-\rho=\frac{R_{0}-\rho_{0}}{2^{j+1}}$, we obtain

$$
A_{j+1} \leq\left[\frac{c \theta_{j}^{2} 2^{j} \mathcal{A}^{\frac{1}{2 n}}}{R_{0}-\rho_{0}}\right]^{\frac{1}{\theta_{j}}} A_{j}
$$

By iteration we get

$$
A_{j+1} \leq\left(\frac{c \mathcal{A}^{\frac{1}{2 n}}}{R_{0}-\rho_{0}}\right)^{\sum_{k=0}^{j} \frac{1}{\theta_{k}}}\left(\prod_{k=0}^{j} \theta_{k}^{\frac{1}{\theta_{k}}}\right)^{2} 2^{\sum_{k=0}^{j} \frac{k}{\theta_{k}}} A_{0}
$$

(observe that $\mathcal{A}$ and $A_{0}$ are finite, thus every $A_{j}$ is finite). The product is finite and the series in the exponents converge and after some calculation, using the definition (3.27) since

$$
\sum_{k=0}^{\infty} \frac{1}{\theta_{k}}=\sum_{k=0}^{\infty}\left(\frac{1^{*} 2}{2^{*}}\right)^{k}=n-1,
$$


and

by the definition of $\mathcal{A}$ we finally have

$$
1+|D u|^{1^{*} 2} g_{t t}(x,|D u|) \leq\left[1+|D u|^{1^{*}} 2 g_{t t}(x,|D u|)\right]^{\frac{2^{*}}{1^{*} 2}},
$$

$$
A_{j+1} \leq \frac{c}{\left(R_{0}-\rho_{0}\right)^{n-1}}\left\{\int_{B_{R_{0}}}\left[1+|D u|^{1^{*}} g_{t t}(x,|D u|)\right]^{\frac{2^{*}}{1^{* 2}}} \mathrm{~d} x\right\}^{\frac{1}{1^{*}}} .
$$

We can easily prove that for every $\beta>0$ and $t \geq 0$ there exists a constant $c=c\left(\Omega_{0}\right)$ such that

$$
t^{\beta} \leq c\left[1+t^{\beta} g_{t t}(x, t)\right], \quad \forall t \geq 0, \quad \text { a.e. } x \in \Omega_{0} .
$$

In fact $(2.3,2.4)$ and $\left(H_{2}\right)$ imply that $g_{t t}(x, 1) \geq g\left(x, t_{0}\right)>\lambda>0$ for a.e. $x \in \Omega_{0}$. We can conclude

$$
\begin{aligned}
\sup \left\{|D u(x)|: x \in B_{\rho_{0}}\right\} & =\lim _{j \rightarrow+\infty}\left\{\int_{B_{\rho_{0}}}|D u(x)|^{2^{*} \theta_{j}} \mathrm{~d} x\right\}^{\frac{1}{2^{*} \theta_{j}}} \\
& \leq \lim _{j \rightarrow+\infty}\left\{c \int_{B_{\rho_{j+1}}}\left[1+|D u|^{2^{*} \theta_{j}} g_{t t}(x,|D u|)\right] \mathrm{d} x\right\}^{\frac{1}{2^{*} \theta_{j}}} \\
& \leq \frac{c}{\left(R_{0}-\rho_{0}\right)^{n-1}}\left\{\int_{B_{R_{0}}}\left[1+|D u|^{1^{*}} 2 g_{t t}(x,|D u|)\right]^{\frac{2^{*}}{1^{*}}} \mathrm{~d} x\right\}^{\frac{1}{1^{*}}} .
\end{aligned}
$$

The last inequality implies that $u \in W_{\text {loc }}^{1, \infty}\left(\Omega, \mathbf{R}^{N}\right)$ and Lemma 3.3 is proved.

Lemma 3.4. Let $\left(H_{1}-H_{4}\right)$ and (3.1, 3.2) hold. If $u$ is a local minimizer of (2.1), then there exist $\sigma=\sigma(n)>0$ and $\alpha=\alpha(n)>0$ such that

$$
\int_{B_{\rho}}\left[1+|D u|^{1^{*} 2} g_{t t}(x,|D u|)\right]^{\frac{2^{*}}{1^{* 2}}} \mathrm{~d} x \leq \frac{c}{(R-\rho)^{\alpha}}\left\{\int_{B_{R}}[1+g(x,|D u|)] \mathrm{d} x\right\}^{1^{*}+\sigma},
$$

where $c$ depends on $n, N$ and on the constants in $\left(H_{1}-H_{4}\right)$.

Proof. Consider the inequality (3.21) in the proof of the previous lemma with $\gamma=0$ (i.e. $\Phi=1$ ):

$$
\left\{\int_{\Omega} \eta^{2^{*}}[G(x,|D u|)]^{2^{*}} \mathrm{~d} x\right\}^{\frac{2}{2^{*}}} \leq c \int_{\Omega}\left[\eta^{2}+|D \eta|^{2}\right]\left[1+|D u|^{2} g_{t t}(x,|D u|)\right]\left[1+g_{t}^{2(\alpha-1)}(x,|D u|)\right] \mathrm{d} x .
$$

Let $1<\delta \leq \frac{2^{*}}{1^{*} 2}$ and apply (iii) of Lemma 3.1 with $\beta=\frac{2^{*}}{\delta} \geq 1^{*} 2>2$ :

$$
[G(x, t)]^{2^{*}}=\left[1+\int_{0}^{t} \sqrt{\frac{g_{t}(x, s)}{s}} \mathrm{~d} s\right]^{\frac{2^{*}}{\delta} \delta} \geq c\left[1+t^{\frac{2^{*}}{\delta}} g_{t t}(x, t)\right]^{\delta} \geq c\left[1+t^{1^{*} 2} g_{t t}(x, t)\right]^{\delta} .
$$

Therefore, choosing the test function $\eta$ and $\epsilon$ as in the proof of Lemma 3.3, we obtain

$$
\begin{aligned}
\left\{\int_{B_{\rho}}\left[1+|D u|^{1^{*}} 2 g_{t t}(x,|D u|)\right]^{\delta} \mathrm{d} x\right\}^{\frac{2}{2^{*}}} & \leq \frac{c}{(R-\rho)^{2}} \int_{B_{R}}\left[1+|D u|^{2} g_{t t}(x,|D u|)\right]\left[1+g_{t t}^{\epsilon}(x,|D u|)|D u|^{\epsilon}\right] \mathrm{d} x \\
& \leq \frac{c}{(R-\rho)^{2}} \int_{B_{R}}\left[1+|D u|^{1^{*} 2} g_{t t}(x,|D u|)\right]^{1+\epsilon} \mathrm{d} x
\end{aligned}
$$


Set

$$
V(x)=1+|D u(x)|^{1^{*} 2} g_{t t}(x,|D u(x)|)
$$

equation (3.30) can be written in the form:

$$
\left\{\int_{B_{\rho}} V^{\delta} \mathrm{d} x\right\}^{\frac{2}{2^{*}}} \leq \frac{c}{(R-\rho)^{2}} \int_{B_{R}} V^{1+\epsilon} \mathrm{d} x
$$

We fix $\delta=\frac{2^{*}}{1^{* 2}}>1$ and let $\gamma>\frac{2^{*}}{2}>\delta$. By using Hölder inequality with exponents $\gamma$ and $\frac{\gamma}{\gamma-1}$, from (3.31) we have

$$
\begin{aligned}
\left\{\int_{B_{\rho}} V^{\delta} \mathrm{d} x\right\}^{\frac{2}{2^{*}}} & \leq \frac{c}{(R-\rho)^{2}} \int_{B_{R}} V^{1+\epsilon} \mathrm{d} x \\
& =\frac{c}{(R-\rho)^{2}} \int_{B_{R}} V^{\frac{\delta}{\gamma}} V^{1-\frac{\delta}{\gamma}+\epsilon} \mathrm{d} x \leq \frac{c}{(R-\rho)^{2}}\left\{\int_{B_{R}} V^{\delta} \mathrm{d} x\right\}^{\frac{1}{\gamma}}\left\{\int_{B_{R}} V^{\frac{\gamma-\delta+\epsilon \gamma}{\gamma-1}} \mathrm{~d} x\right\}^{\frac{\gamma-1}{\gamma}}
\end{aligned}
$$

or equivalently

$$
\int_{B_{\rho}} V^{\delta} \mathrm{d} x \leq \frac{c}{(R-\rho)^{2^{*}}}\left\{\int_{B_{R}} V^{\delta} \mathrm{d} x\right\}^{\frac{2^{*}}{2 \gamma}}\left\{\left[\int_{B_{R}} V^{\frac{\gamma-\delta+\epsilon \gamma}{\gamma-1}} \mathrm{~d} x\right]^{\gamma-1}\right\}^{\frac{2^{*}}{2 \gamma}} .
$$

Fixed $R_{0}$ and $\rho_{0}$ as before, we consider $\rho_{j}=R_{0}-\frac{R_{0}-\rho_{0}}{2^{j}}$. We insert $R=\rho_{j}$ and $\rho=\rho_{j-1}$ in (3.32): since $R-\rho=\frac{R_{0}-\rho_{0}}{2^{j}}$, then we obtain

$$
\int_{B_{\rho_{j-1}}} V^{\delta} \mathrm{d} x \leq\left\{\int_{B_{\rho_{j}}} V^{\delta} \mathrm{d} x\right\}^{\frac{2^{*}}{2 \gamma}} \frac{c 2^{2^{*} j}}{\left(R_{0}-\rho_{0}\right)^{2^{*}}}\left\{\left[\int_{B_{R_{0}}} V^{\frac{\gamma-\delta+\epsilon \gamma}{\gamma-1}} \mathrm{~d} x\right]^{\gamma-1}\right\}^{\frac{2^{*}}{2 \gamma}} .
$$

Denote by $A_{j}=\int_{B_{\rho_{j}}} V^{\delta} \mathrm{d} x$ : by (3.1) and Lemma $3.3, A_{j}$ are uniformly bounded with respect to $j$. Thus (3.33) becomes

$$
A_{j-1} \leq A_{j}^{\frac{2^{*}}{2 \gamma}} \frac{c 2^{2^{*} j}}{\left(R_{0}-\rho_{0}\right)^{2^{*}}}\left\{\left[\int_{B_{R_{0}}} V^{\frac{\gamma-\delta+\epsilon \gamma}{\gamma-1}} \mathrm{~d} x\right]^{\gamma-1}\right\}^{\frac{2^{*}}{2 \gamma}}
$$

Iterating:

$$
\begin{aligned}
A_{0} & \leq A_{j}^{\left(\frac{2^{*}}{2 \gamma}\right)^{j}} \prod_{j=1}^{\infty}\left[\frac{c 2^{2^{*} j}}{\left(R_{0}-\rho_{0}\right)^{2^{*}}}\right]^{\left(\frac{2^{*}}{2 \gamma}\right)^{j}}\left\{\left[\int_{B_{R_{0}}} V^{\frac{\gamma-\delta+\epsilon \gamma}{\gamma-1}} \mathrm{~d} x\right]^{\gamma-1}\right\}^{\left(\frac{2^{*}}{2 \gamma}\right)^{j}} \\
& \leq A_{j}^{\left(\frac{2^{*}}{2 \gamma}\right)^{j}} \frac{c}{\left(R_{0}-\rho_{0}\right)^{2^{*} \frac{2^{*}}{2 \gamma-2^{*}}}}\left\{\int_{B_{R_{0}}} V^{\frac{\gamma-\delta+\epsilon \gamma}{\gamma-1}} \mathrm{~d} x\right\}^{(\gamma-1) \frac{2^{*}}{2 \gamma-2^{*}}}
\end{aligned}
$$

since $\sum_{j=1}^{\infty}\left(\frac{2^{*}}{2 \gamma}\right)^{j}=\frac{2^{*}}{2 \gamma-2^{*}}$. Use (ii) of Lemma 3.1 with exponent $1^{*}>1$, i.e.

$$
1+g_{t t}(x, t) t^{*^{*} 2} \leq c[1+g(x, t)]^{1^{*}}
$$


hence, in this case

We can choose $\gamma$ in such way

$$
V^{\frac{\gamma-\delta+\epsilon \gamma}{\gamma-1}} \leq\left\{c[1+g(x,|D u|)]^{1^{*}}\right\}^{\frac{\gamma-\delta+\epsilon \gamma}{\gamma-1}}
$$

$$
1^{*} \frac{\gamma-\delta+\epsilon \gamma}{\gamma-1}=1
$$

Recalling that $\delta=\frac{2^{*}}{1^{*} 2}$, an easy computation gives $\gamma=\frac{2 \delta}{1+\epsilon n}$ and for $\epsilon$ sufficiently small (i.e. $\alpha$ sufficiently close to 1$), \gamma>\frac{2^{*}}{2}$ as required. With this choice of $\gamma$, from (3.34) we infer

$$
\int_{B_{\rho_{0}}} V^{\delta} \mathrm{d} x \leq\left\{\int_{B_{\rho_{j}}} V^{\delta} \mathrm{d} x\right\}^{\left(\frac{2^{*}}{2 \gamma}\right)^{j}} \cdot \frac{c}{\left(R_{0}-\rho_{0}\right)^{\frac{\left(2^{*}\right)^{2}}{2 \gamma-2^{*}}}}\left\{\int_{B_{R_{0}}}[1+g(x,|D u|)] \mathrm{d} x\right\}^{\frac{2^{*}(\gamma-1)}{2 \gamma-2^{*}}}
$$

and letting $j \rightarrow+\infty$, we conclude

$$
\int_{B_{\rho_{0}}}\left[1+|D u|^{1^{*} 2} g_{t t}(x,|D u|)\right]^{\frac{2^{*}}{1^{*} 2}} \mathrm{~d} x \leq \frac{c}{\left(R_{0}-\rho_{0}\right)^{\frac{\left(2^{*}\right)^{2}}{2 \gamma-2^{*}}}}\left\{\int_{B_{R_{0}}}[1+g(x,|D u|)] \mathrm{d} x\right\}^{\frac{2^{*}(\gamma-1)}{2 \gamma-2^{*}}}
$$

and the Lemma is proved with $\alpha(n)=\frac{\left(2^{*}\right)^{2}}{2 \gamma-2^{*}}$ and $\frac{2^{*}(\gamma-1)}{2 \gamma-2^{*}}=1^{*}+\sigma$ with $\sigma>0$.

Remark 3.5. We underline the fact that the constant $c$ in Propositon 3.2 does not depend on $m, M$ and $N$ of (3.1) and (3.2).

Remark 3.6. It is not difficult to check that the result of Propositon 3.2 holds even if we assume $g$ of class $W_{\text {loc }}^{2, \infty}$ with respect to $t$ for a.e. $x \in \Omega$ instead of class $C^{2}$.

\section{Approximation and proof of the Theorem 2.1}

In this section we will prove the estimate (3.5) of Proposition 3.2 for minimizers of our original functional $F$ and then we have to remove the supplementary assumptions (3.1) and (3.2). The main ingredients are an approximation procedure and then a passage to the limit similar to the ones used by Marcellini in Sections 4 and 5 of [15], modified in order to handle the dependence on $x$ of the integrand.

Let $\Omega_{0} \subset \subset \Omega$ and $g$ satisfy $\left(H_{1}-H_{5}\right)$ of Section 2 . We remember that, by $\left(H_{1}\right)$ and $\left(H_{2}\right)$ :

$$
g(x, 0)=g_{t}(x, 0)=0 \quad \text { and } \quad g_{t}(x, 1) \geq g(x, 1) \geq \lambda>0, \quad \text { a.e. } x \in \Omega_{0} .
$$

For $t \in(0,+\infty)$ and $x \in \Omega$, set

$$
a(x, t)=\frac{g_{t}(x, t)}{t}
$$

which is positive, increasing and $a(x, 1) \geq \lambda>0$ a.e. $x \in \Omega_{0}$.

From assumption $\left(H_{1}\right)$, it follows that $a(x, t)>0$ if $t>0$. For every $k \in \mathbb{N}$, let $t_{k}=\frac{1}{k}$ and define the sequence of functions

$$
a^{k}(x, t)= \begin{cases}a\left(x, t_{k}\right) & \text { for } t \in\left[0, t_{k}\right) \\ a(x, t) & \text { for } t \in\left[t_{k}, k\right] \\ a(x, k) & \text { for } t \in(k,+\infty) .\end{cases}
$$

For every $k \in \mathbb{N}, a^{k}(x, t)$ is continuous and increasing with respect to $t$ and satisfies

$$
a(x, t) \leq a^{k}(x, 1)=a(x, 1) \leq \Lambda, \quad \text { a.e. } x \in \Omega_{0}, \quad \forall t \in[0,1] .
$$


Consider the function $g^{k}(x, t)$ given by

$$
g^{k}(x, t)=\int_{0}^{t} a^{k}(x, s) s \mathrm{~d} s, \quad \text { a.e. } x \in \Omega, \quad \forall t \in[0,+\infty) .
$$

By definition, it follows that fixed $k_{0}$, for every $t \in\left[0, k_{0}\right]$ and $k \geq k_{0}$ we have

$$
0 \leq g^{k_{0}}(x, t)-g^{k}(x, t) \leq \frac{1}{2 k_{0}^{2}} a(x, 1), \quad \text { a.e. } x \in \Omega_{0} .
$$

Moreover $g^{k}(x, t)$ converges pointwise to $g(x, t)$ for a.e. $x \in \Omega$ and $t \geq 0$.

Our next goal is to prove that $g^{k}$ satisfies assumptions $\left(H_{1}-H_{4}\right)$ with constants independent of $k$.

Lemma 4.1. Let $g(x, t)$ satisfy $\left(H_{1}-H_{5}\right)$ and let $g^{k}(x, t)$ defined as in (4.3). Then, for every $\Omega_{0} \subset \subset \Omega, g^{k}$ satisfies $\left(H_{1}\right)$ and $\left(H_{2}\right)$ for $k$ sufficiently large, with constants independent of $k$. Moreover:

(i) for every $k \in \mathbb{N}$, there exist $m_{k}$ and $M_{k}>0$ such that

$$
m_{k} \leq \frac{g_{t}^{k}(x, t)}{t} \leq g_{t t}^{k}(x, t) \leq M_{k}
$$

$\forall t>0$ and a.e. $x \in \Omega_{0}$, where $g_{t t}^{k}(x, k)$ denotes the right second derivatives of $g^{k}$;

(ii) there exists a constant $L=L\left(\Omega_{0}\right)$ such that

$$
g^{k}(x, t) \leq L[1+g(x, t)]
$$

$\forall k \in \mathbb{N}, t \geq 0$ and a.e. $x \in \Omega_{0}$

(iii) for every $\alpha>1$ there exists $C_{1}=C_{1}\left(\alpha, \Omega_{0}\right)$ such that

$$
g_{t t}^{k}(x, t) t^{2 \alpha} \leq C_{1}\left[g^{k}(x, t)\right]^{\alpha}
$$

$\forall k \in \mathbb{N}, t \geq 1$ and a.e. $x \in \Omega_{0} ;$

(iv) for every $k \in \mathbb{N}$, there exists a constant $N_{k}$ such that

$$
\left|g_{t x_{s}}^{k}(x, t)\right| \leq N_{k}\left(1+t^{2}\right)^{\frac{1}{2}}
$$

$\forall t \geq 0$ and a.e. $x \in \Omega_{0}$.

For every $\alpha>1$, there exists $C_{2}=C_{2}\left(\alpha, \Omega_{0}\right)$ such that

$$
\left|g_{t x_{s}}^{k}(x, t)\right| \leq C_{2} g_{t}^{k}(x, t)\left[1+\left(g_{t}^{k}\right)^{\alpha-1}(x, t)\right]
$$

$\forall k \in \mathbb{N}, t \geq 0$ and a.e. $x \in \Omega_{0}$.

Proof. Since $g_{t}^{k}(x, t)=a^{k}(x, t) t$ is increasing with respect to $t$, then $g^{k}(x, t)$ is convex with respect to $t$. Moreover $g^{k}(x, t)$ and $g_{t}^{k}(x, t)$ are Carathéodory functions in $\Omega \times[0,+\infty)$ and $g^{k}(x, t)$ is of class $C^{1}$ with respect to $t$. Since

$$
g_{t t}^{k}(x, t)= \begin{cases}a\left(x, t_{k}\right) & \text { for } t \in\left[0, t_{k}\right) \\ g_{t t}(x, t) & \text { for } t \in\left[t_{k}, k\right] \\ a(x, k) & \text { for } t \in(k,+\infty)\end{cases}
$$

we have, taking into account $\left(H_{5}\right)$, that $g^{k}(x, \cdot) \in W_{\text {loc }}^{2, \infty}$ for a.e. $x \in \Omega$. By construction $a^{k}(x, t)=\frac{g_{t}^{k}(x, t)}{t}$ is increasing, thus $\left(H_{1}\right)$ is satisfied. It is very easy to show that $g_{t t}^{k}(x, t) \leq \Lambda^{\prime}$ for a.e. $x \in \Omega_{0}$ and $\forall t \in[0,1]$ with 
$\Lambda^{\prime}$ independent of $k$; moreover, for $k$ sufficiently large $g^{k}\left(x, t_{0}\right)=g\left(x, t_{0}\right)$, thus $\left(H_{2}\right)$ holds. Let us prove (i). Fixed $x \in \Omega_{0}$, since $a^{k}(x, t)=\frac{g_{t}^{k}(x, t)}{t}$ is increasing and from the definition of $t_{k}$ we have

$$
0<m_{k}=\min _{x \in \Omega_{0}} a\left(x, t_{k}\right) \leq a^{k}(x, t)=\frac{g_{t}^{k}(x, t)}{t} \leq g_{t t}^{k}(x, t)
$$

$\forall t>0$. By taking in account $\left(H_{5}\right)$, set

$$
M_{k}=\max \left\{\|a(x, 1)\|_{L^{\infty}\left(\Omega_{0}\right)},\left\|g_{t t}(x, t)\right\|_{L^{\infty}\left(\Omega_{0} \times[1, k]\right)},\|a(x, k)\|_{L^{\infty}\left(\Omega_{0}\right)}\right\},
$$

thus (4.5) holds.

In order to prove (ii) and (iii), let us show that $\forall k \in \mathbb{N}$ and a.e. $x \in \Omega_{0}$ the following inequalities hold:

$$
\begin{aligned}
g(x, 1) & \leq g^{k}(x, 1) \\
g^{k}(x, t) & \leq a(x, 1)+g(x, t) \quad \forall t \in[0,+\infty) ; \\
g^{k}(x, t) & \geq g(x, t) \quad \forall t \in[1, k)
\end{aligned}
$$

If $t \in[0,1]$, it is clear that $a(x, t) \leq a^{k}(x, t) \leq a(x, 1)$. By using (4.1) and (4.3), we obtain

$$
g^{k}(x, 1)=g^{k}(x, 1)-g^{k}(x, 0)=\int_{0}^{1} a^{k}(x, t) t \mathrm{~d} t \geq \int_{0}^{1} a(x, t) t \mathrm{~d} t=g(x, 1)-g(x, 0)=g(x, 1)
$$

and (4.11) is proved. If $t \in[0,1]$ we have

$$
g^{k}(x, t)=\int_{0}^{t} a^{k}(x, s) s \mathrm{~d} s \leq a(x, 1)
$$

If $t \geq 1$ we have

$$
g_{t}^{k}(x, t)=a^{k}(x, t) t \leq a(x, t) t=g_{t}(x, t)
$$

and thus $\forall t \in[0,+\infty)$

$$
g^{k}(x, t)=g^{k}(x, 1)+\int_{1}^{t} a^{k}(x, s) s \mathrm{~d} s \leq a(x, 1)+g(x, 1)+\int_{1}^{t} a(x, s) s \mathrm{~d} s=a(x, 1)+g(x, t)
$$

and (4.12) is proved. By collecting (4.11) and (4.12), we have

$$
g^{k}(x, t) \leq 2[1+a(x, 1)][1+g(x, t)]
$$

which implies (ii) since $1+a(x, 1)=1+g_{t}(x, 1) \leq 1+\Lambda\left(\Omega_{0}\right)=L$.

In order to prove (4.13) we observe that if $t \in[1, k)$, by (4.11), we have

$$
g^{k}(x, t)=\int_{0}^{1} a^{k}(x, s) s \mathrm{~d} s+\int_{1}^{t} a(x, s) s \mathrm{~d} s=g^{k}(x, 1)+g(x, t)-g(x, 1) \geq g(x, t) .
$$

Let us prove (iii): when $t \in[1, k]$ we use $\left(H_{3}\right)$ and (4.13)

$$
g_{t t}^{k}(x, t) t^{2 \alpha}=g_{t t}(x, t) t^{2 \alpha} \leq c[g(x, t)]^{\alpha} \leq c\left[g^{k}(x, t)\right]^{\alpha},
$$


while for $t \in(k,+\infty)$, by (i) of Lemma 3.1 we have

$$
g_{t t}^{k}(x, t) t^{2 \alpha}=g_{t}(x, k) k^{2 \alpha-1} \frac{t^{2 \alpha}}{k^{2 \alpha}} \leq c[g(x, k)]^{\alpha} \frac{t^{2 \alpha}}{k^{2 \alpha}} .
$$

By proceeding as in the proof of Lemma 4.3 of Marcellini [15], it is possible to show that $g(x, k) \frac{t^{2}}{k^{2}} \leq 2 g^{k}(x, t)$. Thus

$$
g_{t t}^{k}(x, t) t^{2 \alpha} \leq c 2^{\alpha}\left[g^{k}(x, t)\right]^{\alpha},
$$

and (4.7) is proved.

Now we prove (iv). For each fixed $t>0$, the functions $g_{t}^{k}(x, t)$ have weak derivatives with respect to $x_{s}$, $g_{t x_{s}}^{k}(x, t)$, which are Carathéodory functions in $\Omega \times[0,+\infty)$ and locally summable in $\Omega$. If $t \in\left[0, t_{k}\right)$, by $\left(H_{4}\right)$

$$
\begin{aligned}
\left|g_{t x_{s}}^{k}(x, t)\right| & =\left|a_{x_{s}}\left(x, t_{k}\right)\right| t=\left|g_{t x_{s}}\left(x, t_{k}\right) \frac{t}{t_{k}}\right| \leq c_{2} \frac{g_{t}\left(x, t_{k}\right)}{t_{k}} t\left[1+g_{t}^{\alpha-1}\left(x, t_{k}\right)\right] \\
& \leq c_{2} a^{k}(x, t) t\left[1+g_{t}^{\alpha-1}(x, 1)\right] \leq c g_{t}^{k}(x, t)\left[1+\left(g_{t}^{k}\right)^{\alpha-1}(x, t)\right]
\end{aligned}
$$

where $c$ depends on $\Lambda$. If $t \in\left[t_{k}, k\right]$

$$
\left|g_{t x_{s}}^{k}(x, t)\right|=\left|g_{t x_{s}}(x, t)\right| \leq c_{2} g_{t}(x, t)\left[1+g_{t}^{\alpha-1}(x, t)\right] \leq c_{2} g_{t}^{k}(x, t)\left[1+\left(g_{t}^{k}\right)^{\alpha-1}(x, t)\right] .
$$

If $t \in[k,+\infty)$

$$
\left|g_{t x_{s}}^{k}(x, t)\right|=\left|a_{x_{s}}(x, k)\right| t=\left|g_{t x_{s}}(x, k)\right| \frac{t}{k} \leq c_{2} g_{t}(x, k) \frac{t}{k}\left[1+g_{t}^{\alpha-1}(x, k)\right]
$$

thus

$$
\left|g_{t x_{s}}^{k}(x, t)\right| \leq c_{2} g_{t}(x, k) \frac{t}{k}\left[1+\left(\frac{g_{t}(x, k) t}{k}\right)^{\alpha-1}\left(\frac{k}{t}\right)^{\alpha-1}\right] \leq c_{2} g_{t}^{k}(x, t)\left[1+\left(g_{t}^{k}\right)^{\alpha-1}(x, t)\right]
$$

and (4.9) is proved.

Finally, fixed $\alpha_{0}>1$, for $t \in\left[0, t_{k}\right)$,

$$
\left.\left|g_{t x_{s}}^{k}(x, t)\right| \leq c a(x, 1)\left[1+g_{t}^{\alpha_{0}-1}(x, 1)\right]\right] \leq \bar{C},
$$

for $t \in\left[t_{k}, k\right]$,

$$
\left|g_{t x_{s}}^{k}(x, t)\right| \leq c \max _{x \in \Omega_{0}}\left\{g_{t}(x, k)\left[1+g_{t}^{\alpha_{0}-1}(x, k)\right]\right\}=N_{k},
$$

for $t \in[k,+\infty)$, equation (4.14) gives

$$
\left|g_{t x_{s}}^{k}(x, t)\right| \leq c \frac{g_{t}(x, k)}{k} t\left[1+g_{t}^{\alpha_{0}-1}(x, k)\right] \leq \frac{N_{k}}{k}\left(1+t^{2}\right)^{\frac{1}{2}}
$$

and (4.8) holds.

Proof of Theorem 2.1. Let $u$ be a local minimizer of (2.1). For every $k \in \mathbb{N}$ we consider the functional

$$
\int_{\Omega} g^{k}(x,|D u|) \mathrm{d} x
$$


with $g^{k}$ defined as in (4.3). Let $B_{R} \subset \subset \Omega_{0} \subset \subset \Omega$ : the Dirichlet problem

$$
\inf \left\{\int_{B_{R}} g^{k}(x,|D v|) \mathrm{d} x, \quad v \in u+W_{0}^{1,2}\left(B_{R}, \mathbf{R}^{N}\right)\right\}
$$

has one solution $u_{k}$, i.e.

$$
\int_{B_{R}} g^{k}\left(x,\left|D u_{k}\right|\right) \mathrm{d} x \leq \int_{B_{R}} g^{k}(x,|D v|) \mathrm{d} x
$$

for every $v \in u+W_{0}^{1,2}\left(B_{R}, \mathbf{R}^{N}\right)$. In particular

$$
\int_{B_{R}} g^{k}\left(x,\left|D u_{k}\right|\right) \mathrm{d} x \leq \int_{B_{R}} g^{k}(x,|D u|) \mathrm{d} x .
$$

By assumption $\left(H_{3}\right)$ (see (iii) of Lem. 3.1) we have that

$$
t^{2} \leq c\left[1+g^{k}(x, t)\right], \quad \forall t \geq 0, \quad \text { a.e. } x \in \Omega_{0}
$$

and then (4.16) and (4.6) give

$$
\int_{B_{R}}\left|D u_{k}\right|^{2} \mathrm{~d} x \leq c \int_{B_{R}}\left[1+g^{k}(x,|D u|)\right] \mathrm{d} x \leq c \int_{B_{R}}[1+g(x,|D u|)] \mathrm{d} x,
$$

which implies that, up to a subsequence, $\left(u_{k}\right)$ converges weakly in $u+W_{0}^{1,2}\left(B_{R}, \mathbf{R}^{N}\right)$ to a function $w$.

By Lemma 4.1, the functional in (4.15) satisfies the assumptions of Proposition 3.2 and then there exist $\sigma>0$ and $c$ independent on $k$ such that $\forall \rho<R$

$$
\sup _{B_{\rho}}\left|D u_{k}\right| \leq c\left\{\int_{B_{R}}\left[1+g^{k}\left(x,\left|D u_{k}\right|\right)\right] \mathrm{d} x\right\}^{1+\sigma} .
$$

Moreover, by (4.16) and (4.6), we have that for every $k \in \mathbb{N}$

$$
\sup _{B_{\rho}}\left|D u_{k}\right| \leq c\left\{\int_{B_{R}}\left[1+g^{k}(x,|D u|)\right] \mathrm{d} x\right\}^{1+\sigma} \leq c\left\{\int_{B_{R}}[1+g(x,|D u|)] \mathrm{d} x\right\}^{1+\sigma} .
$$

The last inequality gives that $\left(u_{k}\right)$, up to a subsequence, converges to the function $w$ in the weak* topology of $W_{\text {loc }}^{1, \infty}\left(B_{R}, \mathbf{R}^{N}\right)$. Let $k_{0}$ be such that $\left\|D u_{k}\right\|_{L^{\infty}} \leq k_{0}$. By (4.4) and (4.16), we infer that for $k \geq k_{0}$

$$
\int_{B_{\rho}} g^{k_{0}}\left(x,\left|D u_{k}\right|\right) \mathrm{d} x \leq \int_{B_{\rho}} g^{k}\left(x,\left|D u_{k}\right|\right) \mathrm{d} x+\frac{1}{2 k_{0}^{2}} \int_{B_{\rho}} a(x, 1) \mathrm{d} x \leq \int_{B_{R}} g^{k}(x,|D u|) \mathrm{d} x+\frac{c}{k_{0}^{2}} .
$$

By lower semicontinuity and using the dominated convergence theorem, as $k \rightarrow+\infty$ we have

$$
\int_{B_{\rho}} g^{k_{0}}(x,|D w|) \mathrm{d} x \leq \int_{B_{R}} g(x,|D u|) \mathrm{d} x+\frac{c}{k_{0}^{2}}
$$

and then as $k_{0} \rightarrow+\infty$ and $\rho \rightarrow R$ we get

$$
\int_{B_{R}} g(x,|D w|) \mathrm{d} x \leq \int_{B_{R}} g(x,|D u|) \mathrm{d} x .
$$


Therefore $w$ is a local minimizer of $F$ and the strictly convexity of the functional gives $u=w$. Finally (4.17) gives

and thus the theorem is proved.

$$
\|D u\|_{L^{\infty}\left(B_{\rho}, \mathbf{R}^{n N}\right)} \leq c\left\{\int_{B_{R}}[1+g(x,|D u|)] \mathrm{d} x\right\}^{1+\sigma}
$$

\section{REFERENCES}

[1] R. Aris, The mathematical theory of diffusion and reaction of permeable catalysts. Clarendon Press, Oxford (1975).

[2] E. Acerbi and N. Fusco, Regularity for minimizers of non-quadratic functionals: The case $1<p<2$. J. Math. Anal. Appl. 140 (1989) 115-135.

[3] E. Acerbi and G. Mingione, Regularity results for a class of functionals with nonstandard growth. Arch. Rational Mech. Anal. 156 (2001) 121-140.

[4] E. Acerbi and G. Mingione, Regularity results for quasiconvex functionals with nonstandard growth. Ann. Scuola Norm. Sup. Pisa 30 (2001).

[5] V. Chiadò Piat and A. Coscia, Hölder continuity of minimizers of functionals with variable growth exponent. Manuscripta Math. 93 (1997) 283-299.

[6] A. Coscia and G. Mingione, Hölder continuity of the gradient of $p(x)$-harmonic mappings. C. R. Acad. Sci. Paris 328 (1999) 363-368.

[7] A. Dall'Aglio, E. Mascolo and G. Papi, Local boundedness for minima of functionals with non standard growth conditions. Rend. Mat. 18 (1998) 305-326.

[8] A. Dall'Aglio and E. Mascolo, $L^{\infty}$-estimates for a class of nonlinear elliptic systems with non standard growth. Atti Sem. Mat. Fis. Univ. Modena (to appear).

[9] F. Leonetti, E. Mascolo and F. Siepe, Everywhere regularity for a class of vectorial functionals under subquadratic general growth, Preprint. Dipartimento di Matematica "U. Dini", University of Florence.

[10] M. Giaquinta, Multiple integrals in the calculus of variations and non linear elliptic systems. Princeton Univ. Press, Princeton NJ, Ann. Math. Stud. 105 (1983).

[11] M. Giaquinta and G. Modica, Remarks on the regularity of the minimizers of certain degenerate functionals. Manuscripta Math. 57 (1986) 55-99.

[12] E. Giusti, Metodi diretti nel calcolo delle variazioni. UMI, Bologna (1994).

[13] P. Marcellini, Regularity and existence of solutions of elliptic equations with $(p, q)$-growth conditions. J. Differential Equations 90 (1991) 1-30.

[14] P. Marcellini, Regularity for elliptic equations with general growth conditions. J. Differential Equations 105 (1993) $296-333$.

[15] P. Marcellini, Everywhere regularity for a class of elliptic systems without growth conditions. Ann. Scuola Norm. Sup. Pisa 23 (1996) 1-25.

[16] M. Marcus and V.J. Mizel, Continuity of certain Nemitsky operators on Sobolev spaces and chain rule. J. Anal. Math. 28 (1975) 303-334.

[17] E. Mascolo and G. Papi, Local boundedness of integrals of Calculus of Variations. Ann. Mat. Pura Appl. 167 (1994) 323-339.

[18] A.P. Migliorini, Everywhere regularity for a class of elliptic systems with $p, q$ growth conditions. Rend. Istit. Mat. Univ. Trieste XXXI (1999) 203-234.

[19] A.P. Migliorini, Everywhere regularity for a class of elliptic systems with general growth conditions, Ph.D. Thesis. University of Florence, Italy (2000).

[20] J. Mosely, A two dimensional Dirichlet problem with an exponential nonlinearity. SIAM J. Math. Anal. 145 (1983) 719-735.

[21] M. Růžička, Flow of shear dependent electrorheological fluids. C. R. Acad. Sci. Paris 329 (1999) 393-398.

[22] K.R. Rajagopal and M. Růžička, On the modeling of electrorheological materials. Mech. Res. Commun. 23 (1996) $401-407$.

[23] K. Uhlenbeck, Regularity for a class of non-linear elliptic systems. Acta Math. 138 (1977) 219-240.

[24] V.V. ZhiKov, On Lavrentiev phenomenon. Russian J. Math. Phys. 3 (1995) 249-269. 